\title{
Multi-particle systems in quantum spacetime and a novel challenge for center-of-mass motion
}

\author{
Giovanni Amelino-Camelia, ${ }^{1}$ Valerio Astuti, ${ }^{2,3}$ Michelangelo Palmisano, ${ }^{2,3}$ and Michele Ronco ${ }^{4,5}$ \\ ${ }^{1}$ Dipartimento di Fisica Ettore Pancini, Università di Napoli "Federico II", and INFN, \\ Sezione di Napoli, Complesso Univ. Monte S. Angelo, I-80126 Napoli, Italy \\ ${ }^{2}$ Dipartimento di Fisica, Università di Roma "La Sapienza", P.le A. Moro 2, 00185 Roma, Italy \\ ${ }^{3}$ INFN, Sez. Roma1, P.le A. Moro 2, 00185 Roma, Italy \\ ${ }^{4}$ Laboratoire de Physiqe Nucléaire et de Hautes Energies (LPNHE) UPMC, \\ Case courrier 200, 4 place Jussieu, F-75005 Paris, France \\ ${ }^{5}$ UPMC, Case courrier 200, 4 place Jussieu, F-75005 Paris, France
}

\begin{abstract}
In recent times there has been considerable interest in scenarios for quantum gravity in which particle kinematics is affected nonlinearly by the Planck scale, with encouraging results for the phenomenological prospects, but also some concerns that the nonlinearities might produce pathological properties for composite/multiparticle systems. We here focus on kinematics in the $\kappa$-Minkowski noncommutative spacetime, the quantum spacetime which has been most studied from this perspective, and compare the implications of the alternative descriptions of the total momentum of a multiparticle system which have been so far proposed. We provide evidence suggesting that priority should be given to defining the total momentum as the standard linear sum of the momenta of the particles composing the system. We also uncover a previously unnoticed feature concerning some (minute but conceptually important) effects on center-of-mass motion due to properties of the motion of the constituents relative to the center of mass.
\end{abstract}

\section{INTRODUCTION}

Various semi-heuristic arguments [1-3] suggest that the Planck scale should affect relativistic kinematics by introducing some nonlinearities. This is also found deductively in some of the formalisms under consideration in quantum-gravity research, such as the $\kappa$-Minkowski noncommutative spacetime [4, 6] and some models formulated within the "relative-locality framework" 7, 8]. Also noteworthy is the fact that in $2+1 \mathrm{D}$ quantum gravity the Planck scale does indeed introduce some nonlinearities in relativistic kinematics [9]. Further reasons of interest in this possibility come from the phenomenology side: while most other conjectured Planck-scale effects are expected to remain untestable for the foreseeable future, it happens to be the case that nonlinear deformations of relativistic kinematics, even when introduced with Planck-scale suppression, are within the reach of some present and forthcoming experiments and observations [10]. However, due to the complexity of the relevant formalisms, it is not fully established whether models with nonlinear deformations of relativistic kinematics truly are a viable possibility for consistent physics: for systems of only a small number of particles the phenomenological consequences are plausible and interestingly within reach, but for systems composed of a large number of particles there are legitimate concerns 11 13] that the nonlinearities, although minute for each individual particle composing the system, could somehow add up to levels that might be in clear conflict with established experimental facts.

We here explore some of these issues through an analysis of multi-particle systems in the $\kappa$-Minkowski spacetime, a $3+1$-dimensional spacetime whose key property is the non-commutativity of its coordinates:

$$
\left[x_{i}, x_{0}\right]=i \ell x_{i}, \quad\left[x_{i}, x_{j}\right]=0,
$$

where $x_{0}$ is the time, $x_{i}$ are the three spatial coordinates and $\ell$ is a deformation parameter usually assumed to be of the order of the Planck length. A large literature has been devoted to the fact that the symmetries of this quantum space-time are described by the $\kappa$-Poincaré Hopf Algebra which is a non-linear deformation of the standard Poincaré Lie Algebra. Of potential relevance for the description of multiparticle systems in $\kappa$-Minkowski is the observation that, as reviewed in the next section, the structure of the $\kappa$-Poincaré algebra provides room for a nonlinear deformation of the law of composition of momenta. Several studies (see, e.g. 10, 11, 13, 14]) have compared descriptions of total momentum in $\kappa$-Minkowski based on such nonlinear laws of composition to the standard one based on the linear composition of momenta. The main element of novelty we here contribute to this debate concerns the needed consistency between the notion of total momentum and the notion of center-of-mass position coordinates. Some valuable insight is gained when this observation is combined with the requirement that the description of macroscopic systems (systems with a very large number of composing particles) should not be pathological: nonlinearities in the definition of total momentum are in general admissible, but only if they do not grow pathologically for macroscopic systems. Our findings suggest that priority should be given to defining the total momentum as the standard linear sum of the momenta of the particles composing the system. 
Our investigations also uncover a feature which had previously gone unnoticed: in $\kappa$-Minkowski the relative motion and the center of mass motion do not fully decouple. While this feature appears likely to produce only minute (untestably small) effects, it could be rather significant conceptually and it does not appear to be a peculiarity of $\kappa$-Minkowski: it may well be present in other quantum spacetimes, in which however techniques suitable for exposing it have not yet been developed.

In the next section we briefly review some relevant properties of the $\kappa$-Poincaré Hopf algebra. In section III we show how the nonlinear $\kappa$-Poincaré-inspired definition of total momentum leads to a pathological description of macroscopic bodies. In Section IV we propose a new symmetry algebra for the composite system, and expose some advantages of the standard linear definition of total momentum. Some closing remarks are offered in Section $\mathrm{V}$

\section{THE $k$-POINCARÉ ALGEBRA}

We shall adopt the most common description of $\kappa$-Poincaré Hopf algebra which is based on the so called bicross product basis, such that the commutation relations among algebra generators take the following form

$$
\begin{array}{ll}
{\left[P_{\mu}, P_{\nu}\right]=0,} & {\left[R_{i}, P_{0}\right]=0,} \\
{\left[R_{i}, P_{j}\right]=i \epsilon_{i j k} P_{k},} & {\left[R_{i}, R_{j}\right]=i \epsilon_{i j k} R_{k},} \\
{\left[R_{i}, N_{j}\right]=-i \epsilon_{i j k} N_{k},} & {\left[N_{i}, P_{0}\right]=i P_{i},} \\
{\left[N_{i}, P_{j}\right]=i \delta_{i j}\left(\frac{1-e^{-2 \ell P_{0}}}{2 \ell}+\frac{\ell}{2}|\vec{P}|^{2}\right)-i \ell P_{i} P_{j},} & {\left[N_{i}, N_{j}\right]=-i \epsilon_{i j k} R_{k},}
\end{array}
$$

where $R_{i}$ are rotation generators, $N_{i}$ are boost generators, and $P_{\mu}$ are translation generators. These also imply a deformaton of the mass Casimir

$$
m^{2}=\left(\frac{2}{\ell} \sinh \frac{\ell}{2} P_{0}\right)^{2}-e^{\ell P_{0}}|\vec{P}|^{2},
$$

which indeed is an invariant of the symmetry algebra (2).

The fact that in the standard Poincarè Lie algebra the action of generators on products of functions is governed by the Leibniz rule $(X \triangleright(f g)=(X \triangleright f) g+f(X \triangleright g))$ can be expressed in the language of Hopf algebras by stating that the coproducts are primitive:

$$
\begin{aligned}
& \Delta P_{0}=P_{0} \otimes 1+1 \otimes P_{0} \\
& \Delta P_{i}=P_{i} \otimes 1+1 \otimes P_{i} \\
& \Delta N_{i}=N_{i} \otimes 1+1 \otimes N_{i} \\
& \Delta R_{i}=R_{i} \otimes 1+1 \otimes R_{i} .
\end{aligned}
$$

It is well established [4] that for the generators of the $\kappa$-Poincaré Hopf algebra one must adopt deformations of the Leibniz rule, those codified in the following non-primitive coproducts:

$$
\begin{aligned}
& \Delta P_{0}=P_{0} \otimes 1+1 \otimes P_{0} \\
& \Delta P_{i}=P_{i} \otimes 1+e^{-\ell P_{0}} \otimes P_{i} \\
& \Delta N_{i}=N_{i} \otimes 1+e^{-\ell P_{0}} \otimes N_{i}+\ell \epsilon_{i j k} P_{j} \otimes R_{k} \\
& \Delta R_{i}=R_{i} \otimes 1+1 \otimes R_{i} .
\end{aligned}
$$

Such that, for example, $P_{i} \triangleright(f(x) g(x))=\left(P_{i} \triangleright f(x)\right) g(x)+\left(e^{-\ell P_{0}} \triangleright f(x)\right)\left(P_{i} \triangleright g(x)\right)$.

The coproducts of translation generators indeed suggest a nonlinear law of composition of momenta, which for example, in the case of two particles with momenta $p$ and $k$ gives

$$
(p \oplus k)_{0}=p_{0}+k_{0} \quad(p \oplus k)_{i}=p_{i}+e^{-\ell p_{0}} k_{i} .
$$

However, while for microscopic particles $p_{0} \ll 1 / \ell$ (if $\ell$ is indeed of the order of the Planck length), if the two momenta being composed are momenta of macroscopic bodies this composition law is evidently pathological. This shall be a key observation for our description of multiparticle systems.

Also relevant for our investigations is the well-established fact that one can describe the symmetries of $\kappa$-Minkowski in terms of the $\kappa$-Poincaré Hopf algebra [4]. For example, from the following rules of action of the translation generators on coordinates:

$$
P_{0} \triangleright x_{0}=i, \quad P_{i} \triangleright x_{j}=-i \delta_{i j} .
$$


one finds that the $\kappa$-Minkowski commutation relations are covariant:

$$
\begin{gathered}
P_{i} \triangleright\left[x_{j}, x_{0}\right]=P_{i} \triangleright x_{j} x_{0}-P_{i} \triangleright x_{0} x_{j}= \\
=-i \delta_{i j} x_{0}-\left(e^{-\ell P_{0}} \triangleright x_{0}\right) P_{i} \triangleright x_{j}=-i \delta_{i j} x_{0}+i \delta_{i j}\left(1-\ell P_{0}\right) \triangleright x_{0} \\
=\ell \delta_{i j}=i \ell P_{i} \triangleright x_{j}
\end{gathered}
$$

In what here follows important roles are played by the associated deformed Heisenberg algebra [5],

$$
\begin{gathered}
{\left[P_{0}, x_{0}\right]=i, \quad\left[P_{0}, x_{j}\right]=0,} \\
{\left[P_{i}, x_{0}\right]=-i \ell P_{i}, \quad\left[P_{i}, x_{j}\right]=-i \delta_{i j},}
\end{gathered}
$$

and by the representation of boost generators $N_{i}$,

$$
N_{i}=x_{i}\left(\frac{1-e^{-2 \ell P_{0}}}{2 \ell}+\frac{\ell}{2}|\vec{P}|^{2}\right)-x_{0} P_{i}
$$

\section{TROUBLESOME DEFORMATION OF CENTER OF MASS COORDINATES FROM DEFORMATION OF MOMENTUM-COMPOSITION LAW}

Previous studies have focused on the fact that descriptions of total momentum based on the $k$-Poincaré-deformed composition law could produce a paradoxical description of the total momentum of macroscopic bodies. We here intend to notice that descriptions of total momentum based on the $k$-Poincaré-deformed composition law also lead to an unsatisfactory description of the center-of-mass coordinates, an issue which will play a key role in our following observations.

For simplicity we work in a $1+1$-dimensional $k$-Minkowski and focus on only two identical particles $A$ and $B$, describing their total momentum in terms of the $k$-Poincaré-deformed composition law:

$$
\begin{aligned}
& P_{0}^{T}=P_{0}^{A}+P_{0}^{B}, \\
& P_{1}^{T}=P_{1}^{A}+e^{-\ell P_{0}^{A}} P_{1}^{B} .
\end{aligned}
$$

Our requirement is that there should be a pair of coordinates, $x_{0}^{T}, x_{1}^{T}$, describing the position of the center of mass of the system. We expect to have that $P_{\mu}^{T}$ and $x_{\mu}^{T}$ are dual, i.e. they close a (possibly deformed) Heisenberg Algebra. The standard choice of center-of-mass coordinates:

$$
\begin{aligned}
& x_{0}^{T}=\frac{x_{0}^{A}+x_{0}^{B}}{2}, \\
& x_{1}^{T}=\frac{x_{1}^{A}+x_{1}^{B}}{2}
\end{aligned}
$$

is clearly not suitable for ([8]), since one has that:

$$
\begin{aligned}
{\left[P_{1}^{T}, x_{1}^{T}\right] } & =\left[P_{1}^{A}+e^{-\ell P_{0}^{A}} P_{1}^{B}, \frac{x_{1}^{A}+x_{1}^{B}}{2}\right] \\
& =-\frac{i}{2}\left(1+e^{-\ell P_{0}^{A}}\right)
\end{aligned}
$$

and therefore there is no closed (however deformed) center-of-mass Heisenberg algebra.

It is easy to show that a modified definition of the center-of-mass coordinates governed by a closed (deformed) center-of-mass Heisenberg algebra can be found in the form:

$$
\begin{aligned}
& x_{0}^{T}=\frac{x_{0}^{A}+x_{0}^{B}+\frac{\ell}{2}\left(x_{1}^{A} P_{1}^{A}+P_{1}^{A} x_{1}^{A}\right)}{2}, \\
& x_{1}^{T}=\frac{x_{1}^{A}+e^{\ell P_{0}^{A}} x_{1}^{B}}{2}
\end{aligned}
$$


The potential relevance of these coordinates could also be suggested by the fact that they are still $k$-Minkowski coordinates, like those of the constituent particles:

$$
\begin{aligned}
{\left[x_{1}^{T}, x_{0}^{T}\right] } & =\frac{1}{4}\left[x_{1}^{A}, x_{0}^{A}\right]+\frac{1}{4}\left[x_{1}^{A}, \ell x_{1}^{A} P_{1}^{A}\right]+\frac{1}{4}\left[e^{\ell P_{0}^{A}} x_{1}^{B}, x_{0}^{A}\right]+\frac{1}{4}\left[e^{\ell P_{0}^{A}} x_{1}^{B}, x_{0}^{B}\right] \\
& =i \frac{\ell}{4} x_{1}^{A}+i \frac{\ell}{4} x_{1}^{A}+i \frac{\ell}{4} e^{\ell P_{0}^{A}} x_{1}^{B}+i \frac{\ell}{4} e^{\ell P_{0}^{A}} x_{1}^{B} \\
& =i \ell\left(\frac{x_{1}^{A}+e^{\ell P_{0}^{A}} x_{1}^{B}}{2}\right) \\
& =i \ell x_{1}^{T}
\end{aligned}
$$

These $\left\{x_{1}^{T}, x_{0}^{T}\right\}$ together with $\left\{P_{1}^{T}, P_{0}^{T}\right\}$ also satisfy the $k$-deformed Heisenberg algebra:

$$
\begin{array}{ll}
{\left[P_{0}^{T}, x_{0}^{T}\right]=i,} & {\left[P_{0}^{T}, x_{1}^{T}\right]=0,} \\
{\left[P_{1}^{T}, x_{0}^{T}\right]=-i \ell P_{1}^{T},} & {\left[P_{1}^{T}, x_{1}^{T}\right]=-i,}
\end{array}
$$

While all these might be technically reassuring, the emerging physical picture is not encouraging: if these were two macroscopic bodies one would get an unsatisfactory picture not only for the total momentum specified by (8) but also for the spatial coordinates of the center of mass: if $P_{0}^{A} \gg 1 / \ell$ (not uncommon for a macroscopic body if $\ell$ is of the order of the Planck length) then $x_{1}^{T}=\frac{x_{1}^{A}+e^{\ell P_{0}^{A}} x_{1}^{B}}{2}$ would assign a $x_{1}$ coordinate to the center of mass which either is very close to $x_{1}^{A}$, ignoring the $x_{1}^{B}$ contribution completely (if $\ell<0$ ), or is close to being proportional to $x_{1}^{B}$, ignoring the $x_{1}^{A}$ contribution completely (if $\ell>0$ ).

\section{SYMMETRY ALGEBRA OF A SYSTEM OF 2 IDENTICAL PARTICLES}

One our way to an alternative picture, let us then reconsider two particles $A$ and $B$ of equal mass $m$ and coordinates $x_{\mu}^{A}$ and $x_{\mu}^{B}$ satisfying $(\mu \in\{0,1\})$

$$
\left[x_{1}^{A, B}, x_{0}^{A, B}\right]=i \ell x_{1}^{A, B}, \quad\left[x_{\mu}^{A}, x_{\nu}^{B}\right]=0 .
$$

For both of them we can build a $\kappa$-Poincaré algebra with generators $\left\{P_{\mu}^{A, B}, N^{A, B}\right\}$, where in particular the translation generators $P_{\mu}^{A, B}$ act on the respective sets of plane waves as

$$
P_{\mu}^{A, B} \triangleright e^{i k_{1}^{A, B} x_{1}^{A, B}} e^{-i k_{0}^{A, B} x_{0}^{A, B}}=k_{\mu}^{A, B} e^{i k_{1}^{A, B} x_{1}^{A, B}} e^{-i k_{0}^{A, B} x_{0}^{A, B}} .
$$

In other words the plane waves $e^{i k_{1}^{A} x_{1}^{A}} e^{-i k_{0}^{A} x_{0}^{A}}$ and $e^{i k_{1}^{B} x_{1}^{B}} e^{-i k_{0}^{B} x_{0}^{B}}$ diagonalize the action of the operators $P_{\mu}^{A}$ and $P_{\mu}^{B}$, respectively. As in the standard relativistic case, we would like to describe our two-particle system by separating it in a "total" part and a "relative" part. To do so let us recall the undeformed center-of-mass coordinates

$$
x_{\mu}^{T}=\frac{x_{\mu}^{A}+x_{\mu}^{B}}{2}
$$

and the relative ones

$$
x_{\mu}^{R}=\frac{x_{\mu}^{A}-x_{\mu}^{B}}{2} .
$$

It is evidently noteworthy that these center-of-mass coordinates satisfy a $2 \kappa$-Minkowski commutation relation

$$
\left[x_{1}^{T}, x_{0}^{T}\right]=\frac{1}{4}\left(\left[x_{1}^{A}, x_{0}^{A}\right]+\left[x_{1}^{B}, x_{0}^{B}\right]\right)=i \frac{\ell}{2} x_{1}^{T} .
$$

Unlike the case of the center-of-mass coordinates conjugated with the $\kappa$-deformed total momentum, these center-of-mass coordinates give rise to a truly reassuring picture, in which our composite system composed of two particles is governed by a halved deformation parameter. In this way as the number of particles composing the system grows, the deformation effects should become weaker and weaker, thus recovering the standard Poincaré/Galilei physics on macroscopic scales. The only challenges reside in the interface between center-of-mass 
degrees of freedom and degreees of freedom of the motion relative to the center of mass, as one can start to appreciate by noticing that

$$
\begin{array}{rlrl}
{\left[x_{1}^{T}, x_{0}^{T}\right]} & =i \frac{\ell}{2} x_{1}^{T}, & {\left[x_{1}^{R}, x_{0}^{T}\right]=i \frac{\ell}{2} x_{1}^{R},} \\
{\left[x_{1}^{T}, x_{0}^{R}\right]=i \frac{\ell}{2} x_{1}^{R},} & {\left[x_{1}^{R}, x_{0}^{R}\right]=i \frac{\ell}{2} x_{1}^{T} .}
\end{array}
$$

This is further exposed by studying the generators for center-of-mass and relative-motion symmetries which can be paired with these coordinates. En route to those we consider the product of two plane waves, one of "type $A$ " and one of "type B":

$$
e^{i k_{1}^{A} x_{1}^{A}} e^{-i k_{0}^{A} x_{0}^{A}} e^{i k_{1}^{B} x_{1}^{B}} e^{-i k_{0}^{B} x_{0}^{B}}
$$

which we rewrite using the relationship between the coordinates $x_{\mu}^{A, B}$ and the coordinates $x_{\mu}^{T, R}$, finding

$$
e^{i k_{1}^{T} x_{1}^{T}} e^{i k_{1}^{R} x_{1}^{R}} e^{-i k_{0}^{T} x_{0}^{T}} e^{-i k_{0}^{R} x_{0}^{R}}
$$

with

$$
k_{\mu}^{T}=k_{\mu}^{A}+k_{\mu}^{B}, \quad k_{\mu}^{R}=k_{\mu}^{A}-k_{\mu}^{B} .
$$

It is therefore natural to adopt the following intuitive (standard) definition of translation generators

$$
P_{\mu}^{T}=P_{\mu}^{A}+P_{\mu}^{B}, \quad P_{\mu}^{R}=P_{\mu}^{A}-P_{\mu}^{B}
$$

for which one interestingly finds that

$$
P_{\mu}^{T, R} \triangleright e^{i k_{1}^{T} x_{1}^{T}} e^{i k_{1}^{R} x_{1}^{R}} e^{-i k_{0}^{T} x_{0}^{T}} e^{-i k_{0}^{R} x_{0}^{R}}=k_{\mu}^{T, R} e^{i k_{1}^{T} x_{1}^{T}} e^{i k_{1}^{R} x_{1}^{R}} e^{-i k_{0}^{T} x_{0}^{T}} e^{-i k_{0}^{R} x_{0}^{R}},
$$

where we took into account the canonical action of our operators $\left(P_{0} \triangleright x_{0}=i, P_{1} \triangleright x_{1}=-i\right)$.

It is then straightforward to verify that

$$
\begin{aligned}
{\left[P_{0}^{T}, x_{0}^{T}\right] } & =i, & {\left[P_{0}^{T}, x_{0}^{R}\right] } & =0, \\
{\left[P_{0}^{T}, x_{1}^{T}\right] } & =0, & {\left[P_{0}^{T}, x_{1}^{R}\right] } & =0, \\
{\left[P_{0}^{R}, x_{0}^{T}\right] } & =0, & {\left[P_{0}^{R}, x_{0}^{R}\right] } & =i, \\
{\left[P_{0}^{R}, x_{1}^{T}\right] } & =0, & {\left[P_{0}^{R}, x_{1}^{R}\right] } & =0, \\
{\left[P_{1}^{T}, x_{0}^{T}\right] } & =-i \frac{\ell}{2} P_{1}^{T}, & {\left[P_{1}^{T}, x_{0}^{R}\right] } & =-i \frac{\ell}{2} P_{1}^{R}, \\
{\left[P_{1}^{T}, x_{1}^{T}\right] } & =-i, & {\left[P_{1}^{T}, x_{1}^{R}\right] } & =0 \\
{\left[P_{1}^{R}, x_{0}^{T}\right] } & =-i \frac{\ell}{2} P_{1}^{R}, & {\left[P_{1}^{R}, x_{0}^{R}\right] } & =-i \frac{\ell}{2} P_{1}^{T}, \\
{\left[P_{1}^{R}, x_{1}^{T}\right] } & =0, & {\left[P_{1}^{R}, x_{1}^{R}\right] } & =-i
\end{aligned}
$$

and derive the following coproducts

$$
\begin{aligned}
& \Delta\left(P_{0}^{T, R}\right)=P_{0}^{T, R} \otimes 1+1 \otimes P_{0}^{T, R}, \\
& \Delta\left(P_{1}^{T}\right)=P_{1}^{T} \otimes 1+e^{-\frac{\ell}{2} P_{0}^{T}} \cosh \frac{\ell}{2} P_{0}^{R} \otimes P_{1}^{T}-e^{-\frac{\ell}{2} P_{0}^{T}} \sinh \frac{\ell}{2} P_{0}^{R} \otimes P_{1}^{R}, \\
& \Delta\left(P_{1}^{R}\right)=P_{1}^{R} \otimes 1+e^{-\frac{\ell}{2} P_{0}^{T}} \cosh \frac{\ell}{2} P_{0}^{R} \otimes P_{1}^{R}-e^{-\frac{\ell}{2} P_{0}^{T}} \sinh \frac{\ell}{2} P_{0}^{R} \otimes P_{1}^{T} .
\end{aligned}
$$

Notice how the first two coproducts (as well as the relevant commutators in the deformed Heisenberg algebra (25)) produce a picture in which the motion relative to the center of mass does not decouple from the motion of the center of mass itself; however, this conceptually important feature might have little or no observable consequences, since for small energy-momentum of the relative motion the decoupling is restored and for typical macroscopic bodies one does expect small energy-momentum of the relative motion. We find that, when one can neglect the 
energy-momentum of the relative motion, the first two coproducts (as well as the relevant commutators in the deformed Heisenberg algebra (25)) reduce to $2 \kappa$-Poincaré ones.

For what concerns boost generators, we get a promising picture by simply emulating the definition of our total and relative translation generators, i.e.:

$$
\begin{aligned}
& N^{T}=N^{A}+N^{B}, \\
& N^{R}=N^{A}-N^{B} .
\end{aligned}
$$

Using the single particle boost generators representation (7), the equations in (27) can be explicitly written as

$$
N^{T}=x_{1}^{T}\left[\frac{1-e^{-\ell P_{0}^{T}} \cosh \ell P_{0}^{R}}{\ell}+\frac{\ell}{4}\left(P_{1}^{T^{2}}+P_{1}^{R^{2}}\right)\right]+x_{1}^{R}\left(\frac{e^{-\ell P_{0}^{T}} \sinh \ell P_{0}^{R}}{\ell}+\frac{\ell}{2} P_{1}^{T} P_{1}^{R}\right)-x_{0}^{T} P_{1}^{T}-x_{0}^{R} P_{1}^{R} .
$$

and

$$
N^{R}=x_{1}^{T}\left(\frac{e^{-\ell P_{0}^{T}} \sinh \ell P_{0}^{R}}{\ell}+\frac{\ell}{2} P_{1}^{T} P_{1}^{R}\right)+x_{1}^{R}\left[\frac{1-e^{-\ell P_{0}^{T}} \cosh \ell P_{0}^{R}}{\ell}+\frac{\ell}{4}\left(P_{1}^{T^{2}}+P_{1}^{R^{2}}\right)\right]-x_{0}^{T} P_{1}^{R}-x_{0}^{R} P_{1}^{T} .
$$

Since we can easily go from the set of generators $\left\{P_{\mu}^{A, B}, N^{A, B}\right\}$ to the set $\left\{P_{\mu}^{T, R}, N^{T, R}\right\}$ and viceversa, these show that the set $\left\{P_{\mu}^{T, R}, N^{T, R}\right\}$ forms a closed algebra of symmetry. For the commutators between the boost and the time translation generators one straightforwardly finds

$$
\begin{aligned}
& {\left[N^{T}, P_{0}^{T}\right]=\left[N^{A}+N^{B}, P_{0}^{A}+P_{0}^{B}\right]=i P_{1}^{T},} \\
& {\left[N^{T}, P_{0}^{R}\right]=\left[N^{A}+N^{B}, P_{0}^{A}-P_{0}^{B}\right]=i P_{1}^{R},} \\
& {\left[N^{R}, P_{0}^{T}\right]=\left[N^{A}-N^{B}, P_{0}^{A}+P_{0}^{B}\right]=i P_{1}^{R},} \\
& {\left[N^{R}, P_{0}^{R}\right]=\left[N^{A}-N^{B}, P_{0}^{A}-P_{0}^{B}\right]=i P_{1}^{T} .}
\end{aligned}
$$

The derivations of the remaining commutators are a little more involved but still manageable. For instance one has:

$$
\begin{aligned}
{\left[N^{T}, P_{1}^{T}\right] } & =\left[N^{A}, P_{1}^{A}\right]+\left[N^{B}, P_{1}^{B}\right] \\
& =\frac{1-e^{-2 \ell P_{0}^{A}}}{2 \ell}-\frac{\ell}{2} P_{1}^{A^{2}}+\frac{1-e^{-2 \ell P_{0}^{B}}}{2 \ell}-\frac{\ell}{2} P_{1}^{B^{2}} \\
& =\frac{1-\frac{e^{-2 \ell P_{0}^{A}}+e^{-2 \ell P_{0}^{B}}}{2}}{\ell}-\frac{\ell}{2}\left(P_{1}^{A^{2}}+P_{1}^{B^{2}}\right) \\
& =\frac{1-e^{-\ell P_{0}^{T}} \cosh \ell P_{0}^{R}}{\ell}-\frac{\ell}{4}\left(P_{1}^{T^{2}}+P_{1}^{R^{2}}\right) .
\end{aligned}
$$

and in similar fashion:

$$
\begin{aligned}
{\left[N^{T}, P_{1}^{R}\right] } & =\left[N^{A}, P_{1}^{A}\right]-\left[N^{B}, P_{1}^{B}\right] \\
& =\frac{1-e^{-2 \ell P_{0}^{A}}}{2 \ell}-\frac{\ell}{2} P_{1}^{A^{2}}-\frac{1-e^{-2 \ell P_{0}^{B}}}{2 \ell}+\frac{\ell}{2} P_{1}^{B^{2}} \\
& =\frac{-e^{-2 \ell P_{0}^{A}}+e^{-2 \ell P_{0}^{B}}}{2 \ell}-\frac{\ell}{2}\left(P_{1}^{A^{2}}-P_{1}^{B^{2}}\right) \\
& =e^{-\ell P_{0}^{T}} \frac{\sinh \ell P_{0}^{R}}{\ell}-\frac{\ell}{2} P_{1}^{T} P_{1}^{R} .
\end{aligned}
$$

It is easy to realize that the remaining two commutators are actually identical to the previous ones, since they end up connecting the single particle generators $A$ or $B$ with the same signs + or - , that is

$$
\left[N^{R}, P_{1}^{T}\right]=\left[N^{T}, P_{1}^{R}\right], \quad\left[N^{R}, P_{1}^{R}\right]=\left[N^{T}, P_{1}^{T}\right] .
$$

Finally it is not hard to compute the coproducts of the total and relative boost generators:

$$
\begin{aligned}
& \Delta\left(N^{T}\right)=N^{T} \otimes 1+e^{-\frac{\ell}{2} P_{0}^{T}} \cosh \frac{\ell}{2} P_{0}^{R} \otimes N^{T}-e^{-\frac{\ell}{2} P_{0}^{T}} \sinh \frac{\ell}{2} P_{0}^{R} \otimes N^{R} \\
& \Delta\left(N^{R}\right)=N^{R} \otimes 1+e^{-\frac{\ell}{2} P_{0}^{T}} \cosh \frac{\ell}{2} P_{0}^{R} \otimes N^{R}-e^{-\frac{\ell}{2} P_{0}^{T}} \sinh \frac{\ell}{2} P_{0}^{R} \otimes N^{T},
\end{aligned}
$$


which unsurprisingly have the same form of the coproducts (26). Notice again that for small relative momentum, both the commutators and the coproducts tend to the $2 \kappa$-Poincaré ones.

We therefore have a description of the relativistic symmetries of our two-particle system in terms of center-of-mass degrees of freedom and degrees of freedom of the motion relative to the center of mass which is given by a robust Hopf algebra, with generators $\left\{P_{0}^{T}, P_{0}^{R}, P_{1}^{T}, P_{1}^{R}, N^{T}, N^{R}\right\}$. In cases where there is negligible motion relative to the center of mass the relativistic symmetries are fully described by a $2 \kappa$-Poincaré Hopf algebra, with the prefix " $2 \kappa$ " signaling the fact that, denoting with $\ell$ the deformation parameter of the $\kappa$-Poincaré Hopf algebra governing the single-particle case, for the center of mass of a two-particle system we have exactly the same symmetries (if indeed there is negligible motion relative to the center of mass) but with halved deformation parameter, $\frac{\ell}{2}$.

In closing this section we want to provide further (and more explicit) observations concerning the emergence of the $2 \kappa$-Poincaré Hopf algebra. We start by noticing that, in light of results established above, as basis for the space of fields describing two identical particles in $\kappa$-Minkowski one can equivalently adopt either

$$
e^{i k_{1}^{A} x_{1}^{A}} e^{-i k_{0}^{A} x_{0}^{A}} e^{i k_{1}^{B} x_{1}^{B}} e^{-i k_{0}^{B} x_{0}^{B}} .
$$

or

$$
e^{i k_{1}^{T} x_{1}^{T}} e^{i k_{1}^{R} x_{1}^{R}} e^{-i k_{0}^{T} x_{0}^{T}} e^{-i k_{0}^{R} x_{0}^{R}}
$$

Then a generic function describing the two particles can be written as

$$
g\left(x_{1}^{T}, x_{0}^{T}, x_{1}^{R}, x_{0}^{R}\right)=\int d^{2} k^{T} d^{2} k^{R} \tilde{g}\left(k_{1}^{T}, k_{0}^{T}, k_{1}^{R}, k_{0}^{R}\right) e^{i k_{1}^{T} x_{1}^{T}} e^{i k_{1}^{R} x_{1}^{R}} e^{-i k_{0}^{T} x_{0}^{T}} e^{-i k_{0}^{R} x_{0}^{R}} .
$$

The subspace $C^{T}$ of fields which depend exclusively on the center-of-mass coordinates can be characterized by restricting the analysis to functions $\tilde{g}$ such that $\tilde{g}\left(k_{1}^{T}, k_{0}^{T}, k_{1}^{R}, k_{0}^{R}\right)=0$ whenever $k_{1}^{R} \neq 0$ and/or $k_{0}^{R} \neq 0$. On this subspace of fields the relativistic symmetries are fully described by the $2 k$-Poincaré Hopf algebra, and in particular for such fields the boost generator admits the following simple description

$$
N^{T}=x_{1}^{T}\left(\frac{1-e^{-\ell P_{0}^{T}}}{\ell}+\frac{\ell}{4} P_{1}^{T^{2}}\right)-x_{0}^{T} P_{1}^{T} .
$$

\section{SUMMARY AND OUTLOOK}

The study we here reported intends to contribute to the search of the proper description of multi-particle systems in some quantum spacetimes, using the $\kappa$-Minkowski spacetime as reference example. We obtained new results for both of the most studied definitions of total momentum in $\kappa$-Minkowski. In particular, we observed that the definition of total momentum which is inspired by the $\kappa$-Poincaré coproduct is even more concerning than previously appreciated: we found that besides being, as already known, based on nonlinearities that could be pathological for macroscopic bodies, it also leads to a paradoxical description of center-of-mass coordinates and/or a picture in which there is no closed (deformed) Heisenberg algebra for the center-of-mass motion. The results we obtained for the alternative undeformed definition of total momentum appear to be more encouraging: not only the total momentum does not involve nonlinearities but also the full description of relativistic symmetries appears to be well behaved as the number of particles in the system grows (signaled in our study by the emergence of a $2 \kappa$-Poincarè description for the two-particle system from the $\kappa$-Poincarè description of the composing particles); moreover, one gets a closed (deformed) Heisenberg algebra for the center-of-mass motion. The only residual peculiarity present when adopting the undeformed definition of total momentum resides in an incomplete separation between center-of-mass motion and motion relative to the center of mass, which however does not appear to be alarming since its form would be inconsequential in typical many-particle systems, whose relative motion is strongly suppressed with respect to the center-of-mass motion. Nonetheless, these limitations to the separation between center-of-mass motion and motion relative to the center of mass are noteworthy from a conceptual perspective, and we conjecture that they will be found also in other quantum spacetimes. We feel that some priority should be given to the development of suitable techniques of analysis of this issue adapted to the different quantum spacetimes that might be affected by it. Evidently our findings also provide motivation for further investigation of another long-standing issue for these scenarios with deformed relativistic symmetries, which concerns the possibility of nonuniversal relativistic behavior: if a two-particle composite system is governed by $2 \kappa$-Poincarè relativistic properties while single particles are governed by $\kappa$-Poincarè relativistic properties then the description of processes involving both a two-particle composite system and a fundamental particle should require a sophisticated picture that takes into account the different relativistic properties. Preliminary results suggest that this can be done satisfactorily (see, e.g., Refs. [15, 16]), but more in-depth analyses are needed. 


\section{ACKNOWLEDGEMENTS}

G.A.-C.'s work on this project was supported by the FQXi grant 2018-190483 and by the MIUR, PRIN 2017 grant 20179ZF5KS.

[1] G. Amelino-Camelia, Relativity in space-times with short-distance structure governed by an observer-independent (Planckian) length scale, International Journal of Modern Physics D, Vol. 11, N. 01, pp. 35-59 (2002)

[2] S. Doplicher, K. Fredenhagen, J. E. Roberts, The quantum structure of spacetime at the Planck scale and quantum fields,Communications in Mathematical Physics 172.1: 187-220 (1995)

[3] P.G. Bergmann, G.J. Smith, Measurability analysis of the linearized gravitational field, Gen. Rel. Grav. 14 1131-1166 (1982)

[4] S. Majid, H. Ruegg, Bicrossproduct structure of $\kappa$-Poincaré group and non-commutative geometry, Physics Letters B 334.3-4: 348-354 (1994)

[5] J. Lukierski, H. Ruegg, W.J. Zakrzewski. Classical and quantum mechanics of free א-relativistic systems, Annals of Physics 243.1: 90-116 (1995)

[6] G. Amelino-Camelia, V. Astuti, G. Rosati, Relative locality in a quantum spacetime and the pregeometry of $\kappa$-Minkowski, The European Physical Journal C 73.8: 2521 (2013)

[7] G. Amelino-Camelia, L. Freidel, J. Kowalski-Glikman, L. Smolin, Principle of relative locality, Physical Review D 84.8: $084010(2011)$

[8] G. Gubitosi, F. Mercati, Relative locality in א-Poincaré, Classical and Quantum Gravity 30.14: 145002 (2013)

[9] L. Freidel, E. R. Livine, 3D quantum gravity and effective noncommutative quantum field theory, Physical review letters 96.22: $221301(2006)$

[10] G. Amelino-Camelia, Quantum spacetime phenomenology, Living Rev. Rel. 165 (2013)

[11] G. Amelino-Camelia, L. Freidel, J. Kowalski-Glikman, L. Smolin, Relative locality and the soccer ball problem, Phys.Rev. D84 $087702(2011)$

[12] S. Hossenfelder, The Soccer-Ball Problem, SIGMA 10 (2014)

[13] G. Amelino-Camelia, Planck-Scale Soccer-Ball Problem: A Case of Mistaken Identity, Entropy 19 no.8, 400 (2017)

[14] T. Jacobson, S. Liberati, D. Mattingly, Lorentz violation at high energy: Concepts, phenomena and astrophysical constraints, Ann. Phys. 321, 150-196 (2006)

[15] G. Amelino-Camelia, Particle-Dependent Deformations of Lorentz Symmetry, Symmetry, 4(3), 344-378 (2012)

[16] M. Palmisano, G. Amelino-Camelia, M. Ronco, G. D'Amico, Mixing coproducts for theories with particle-dependent relativistic properties, International Journal of Modern Physics D, Vol. 29, Issue N. 022050017 (2020) 